\title{
Communicable Diseases Report, NSW, March and April 2009
}

\section{Communicable Diseases Branch NSW Department of Health}

For updated information, including data and facts on specific diseases, visit www.health.nsw.gov.au and click on Public Health then Infectious Diseases, or access the site directly at: http://www.health. nsw.gov.au/public health/infectious/index.asp.

Figure 1 and Tables 1 and 2 show reports of communicable diseases received through to the end of April 2009 in New South Wales (NSW).

\section{Respiratory diseases H1N1 influenza 09 (human swine influenza)}

The first cases of human swine influenza were reported in Mexico and North America during April 2009. Subsequent spread to several other countries has prompted the World Health Organization to raise the pandemic alert level to five (out of a possible six). The situation is evolving rapidly and intense public health control measures are in place.

\section{Seasonal influenza}

Seasonal influenza will appear in NSW this winter regardless of whether H1N1 influenza 09 emerges in Australia. The severity of seasonal influenza outbreaks depends on several factors including: how different the circulating strain is to the strains that circulated in previous years (few people in the community will have sufficient immunity to a new strain); how well the strains in the vaccine are matched to the circulating strain; the proportion of the population that is vaccinated; and other characteristics of the virus.

\section{Invasive meningococcal disease}

Thirteen cases of invasive meningococcal disease were reported in March and April in NSW, bringing the total number of cases to 23 so far this year. There were 12 cases in the same period in 2008.

\section{Legionnaires' disease}

Fifteen cases of Legionnaires' disease were notified in March and April in NSW. Of these, eight were due to L. pneumophila infection (acquired from environmental sources such as water droplets), six were due to L. longbeachae (acquired from environmental sources such as soils) and one was not specified. The cases were unrelated. Twenty-six cases of Legionella infection have been notified in NSW so far this year, compared with 89 for the same period in 2008 .

\section{Vaccine-preventable diseases Pertussis (whooping cough)}

The pertussis outbreak continued in NSW with 3069 cases notified with onset in March and April. In 2008, a total of 8851 cases were notified, and in 2007 only 2099 cases were notified. Comparison of data over time must be undertaken with caution, however, due to recent changes in the use of diagnostic technologies (including the increasing use of nucleic acid testing), as well as changes in case ascertainment over time (related to increased awareness of the disease among doctors and the broader community).

A 4-week-old infant from the NSW North Coast died from pertussis in early March. The infant was admitted to hospital a week earlier following onset of the illness. While deaths from pertussis are rare, there were six child fatalities in NSW during an outbreak in the mid-to-late 1990s.

Timely immunisation of infants is important as unvaccinated infants are at highest risk of infection and at high risk of complications. Because pertussis immunity wanes over time, many older children and adults are susceptible to infection and can be the source of new infections in infants.

For a limited time in NSW, free pertussis (dTpa) vaccine will be available for all new parents, couples who are planning a pregnancy, grandparents and any other adults who will regularly care for infants less than 12 months of age.

General practitioners should:

- Check the immunisation status of new parents and other children and provide catch-up vaccination.

- Offer free dTpa vaccine to new parents, grandparents and any other adults who regularly care for infants. 
- Consider vaccinating infants at 6 weeks if the opportunity arises. Infanrix-hexa, Prevenar and Rotarix are all licensed for use in infants from 6 weeks of age. The next scheduled vaccines should be given at 4 and 6 months of age.

\section{Measles}

Two cases of measles were notified in March and April in NSW, bringing the total for the year so far to eight. Thirtynine cases were notified in 2008. The majority of measles cases notified so far this year have been in young people recently returned from overseas travel, or in their contacts.

Many people born between 1966 and 1980 remain susceptible to measles because most people in this age group have not been exposed to measles infection and those who were routinely immunised typically received only one measles vaccine. Two doses are required to provide high level protection. Anyone born after 1965 should ensure that they have had two doses of Measles-Mumps-Rubella vaccine, unless they know they are immune.

\section{Tetanus}

One case of tetanus was notified in an unimmunised overseas traveller, bringing the total for the year to date to two.

While uncommon, tetanus can be fatal. Tetanus vaccine is given at 2, 4 and 6 months of age, with boosting doses at 4 years, between 15 and 17 years, and again at 50 years. In recent years, cases in NSW have tended to be older Australian-born women. A likely explanation for this is that childhood vaccination only became available after World War II and predominantly male military personnel received vaccination during service.

Opportunistically reviewing and updating vaccinations of older Australians and people born in developing countries, who may have missed out on childhood vaccination, as well as managing tetanus-prone wounds in people of all ages contributes to prevention of this disease.

\section{Enteric diseases \\ Cryptosporidiosis}

A large outbreak of cryptosporidiosis peaked in March, with 411 laboratory-confirmed cases notified in NSW. A further 201 reported cases in April bring the year to date total to 612 , which exceeds the total of 484 cases reported in 2008 .

\section{Salmonellosis}

Cases of laboratory-confirmed Salmonella infection were above seasonal expectations in the first 3 months of 2009, with 608 cases notified in March and April in NSW. The largest increases occurred in S. Typhimurium, phage typed as 170. Investigations into some recent outbreaks have suggested the source of infection to be poor handling of contaminated food, including eggs. Raw eggs have previously been linked to multiple outbreaks of salmonellosis.

\section{Shigellosis}

Thirty-eight laboratory-confirmed cases of Shigella infection were notified in March and April in NSW, compared with 14 for the same period in 2008 .

\section{Gastroenteritis}

In March and April, 48 outbreaks of gastroenteritis were notified in institutional settings in NSW, affecting a total of 613 people. Of these, 15 were in aged-care facilities, five in hospitals, 26 in child care centres, one in a school and one in a mental health facility. The majority of these outbreaks appear to have been caused by viral infections, spread by person-to-person transmission.

Clinical specimens were submitted for testing from 17 suspected person-to-person gastroenteritis outbreaks. Norovirus was confirmed in stool samples from patients in five outbreaks. In one outbreak, Clostridium perfringens was considered a probable cause. This number of outbreaks is typical of this time of year.

There were eight suspected foodborne outbreaks in March and April in NSW, affecting 232 people. Of these, six were outbreaks of salmonellosis, one of which was linked to consumption of deep-fried ice cream.

\section{Other conditions \\ Community acquired methicillin-resistant \\ Staphylococcus aureus}

Methicillin-resistant Staphylococcus aureus is an ongoing concern both in health care settings and in the general community.

Many healthy people carry staphylococcal bacteria on their skin and in their nose. Some strains of S. aureus, known as methicillin-resistant Staphylococcus aureus (MRSA), are resistant to methicillin and other antibiotics. Within this group, some strains, known as Community acquired MRSA (CaMRSA), are more likely to spread within the community rather than in health care settings. CaMRSA infections can manifest as pimples and boils, impetigo or cellulitis, osteomyelitis, bacteraemia and pneumonia.

Two new factsheets have been developed to assist clinicians, public health practitioners and others to help manage patients with CaMRSA:

http://www.health.nsw.gov.au/factsheets/infectious/staph_ aureus.html

http://www.health.nsw.gov.au/factsheets/guideline/ methicillin_res_stap.html 
Figure 1. Reports of selected communicable diseases, NSW, January 2004 to April 2009, by month of onset.

Preliminary data: case counts in recent months may increase because of reporting delays.

Laboratory-confirmed cases only, except for measles, meningococcal disease and pertussis.

BFV, Barmah Forest virus infection; RRV, Ross River virus infection; lab conf, laboratory confirmed;

Men $\mathrm{Gp} C$ and $\mathrm{Gp} B$, meningococcal disease due to serogroup $C$ and serogroup B infection;

other/unk, other or unknown serogroups.

NB: Multiple series in graphs are stacked, except gastroenteritis outbreaks.

NB: Outbreaks are more likely to be reported by nursing homes and hospitals than by other institutions.

\begin{tabular}{|lr|}
\hline \multicolumn{2}{|c|}{ NSW Population } \\
Male & $50 \%$ \\
$<5$ y & $7 \%$ \\
$5-24$ y & $27 \%$ \\
$25-64$ y & $53 \%$ \\
$65+y$ & $13 \%$ \\
Rural & $46 \%$ \\
\hline
\end{tabular}

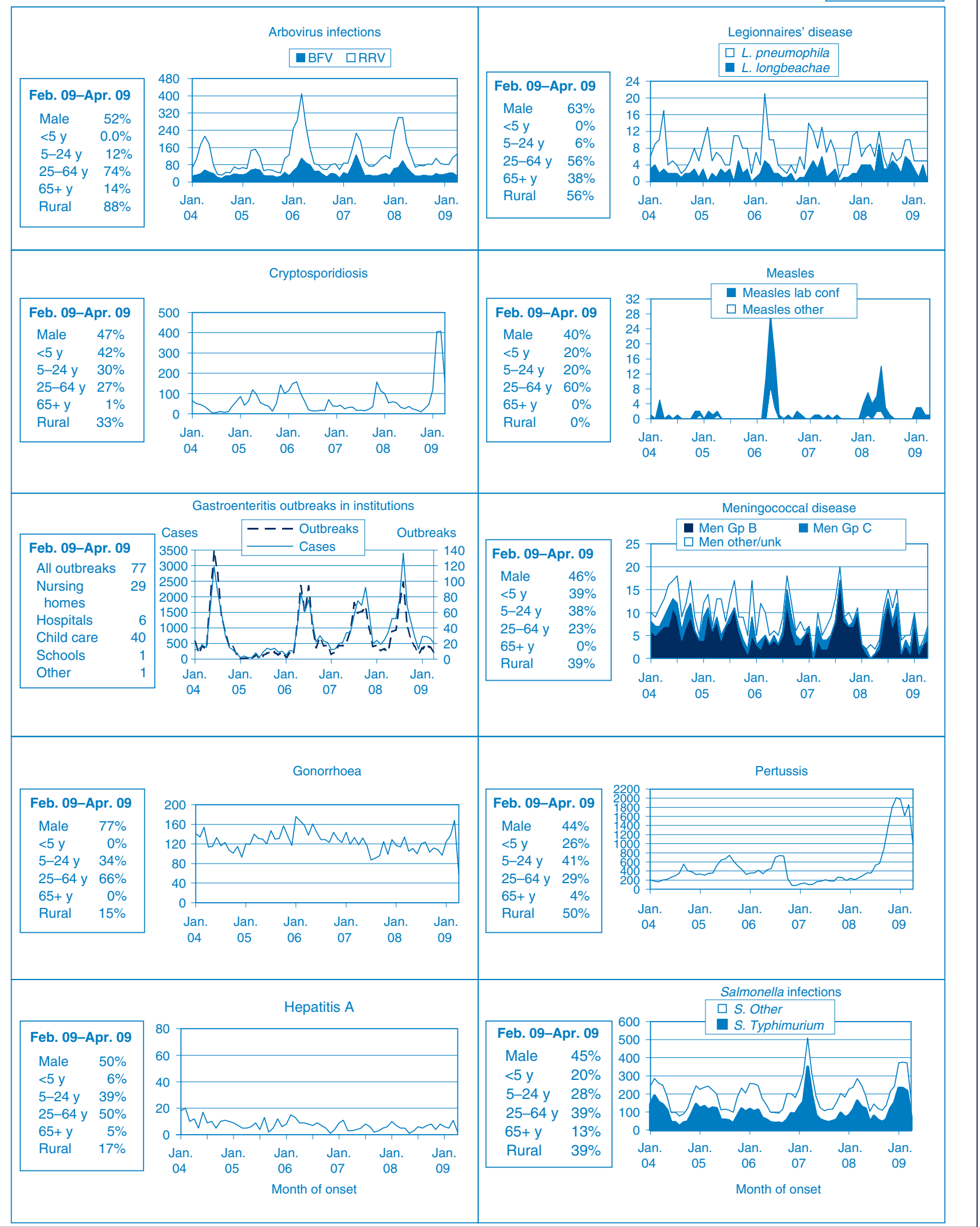




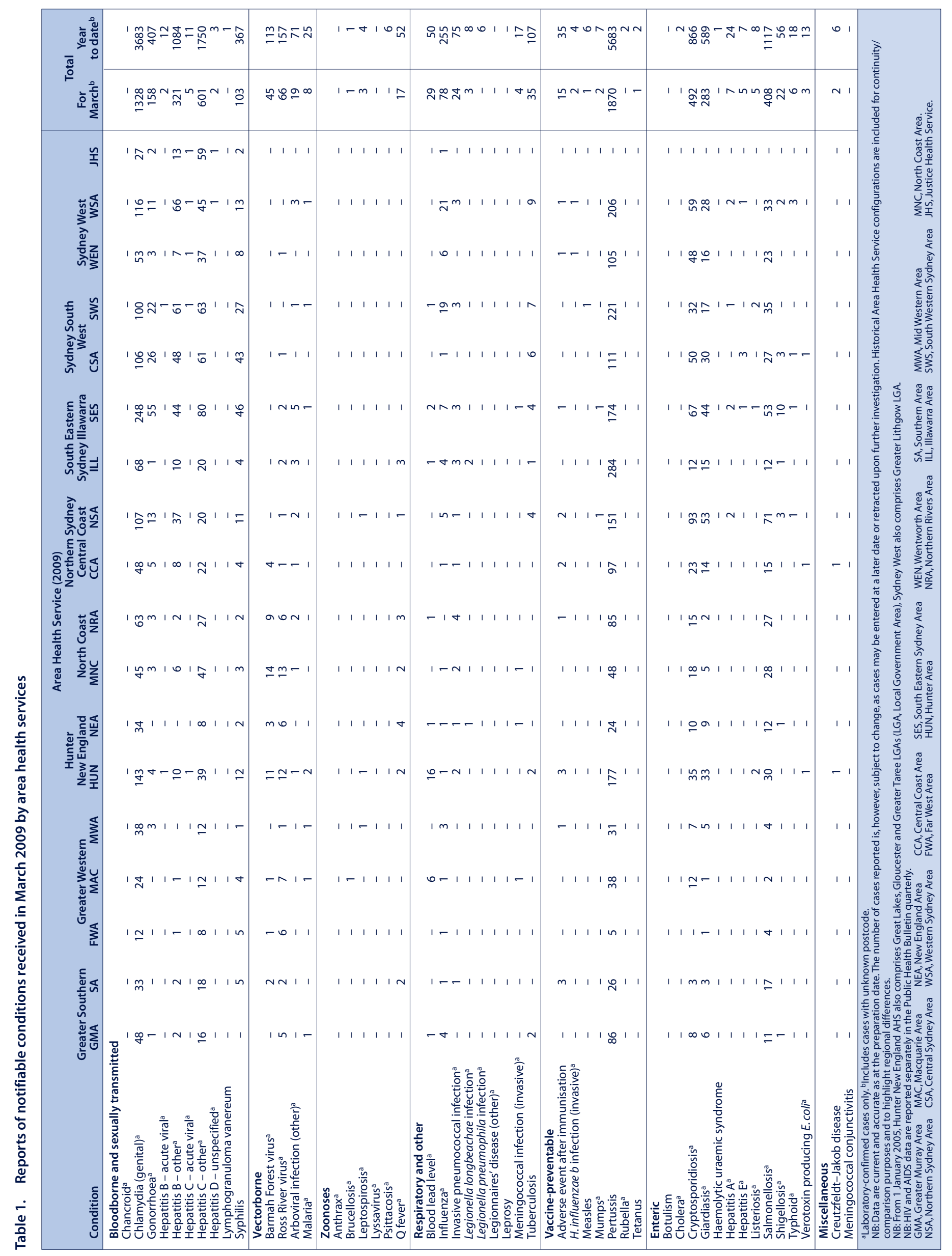




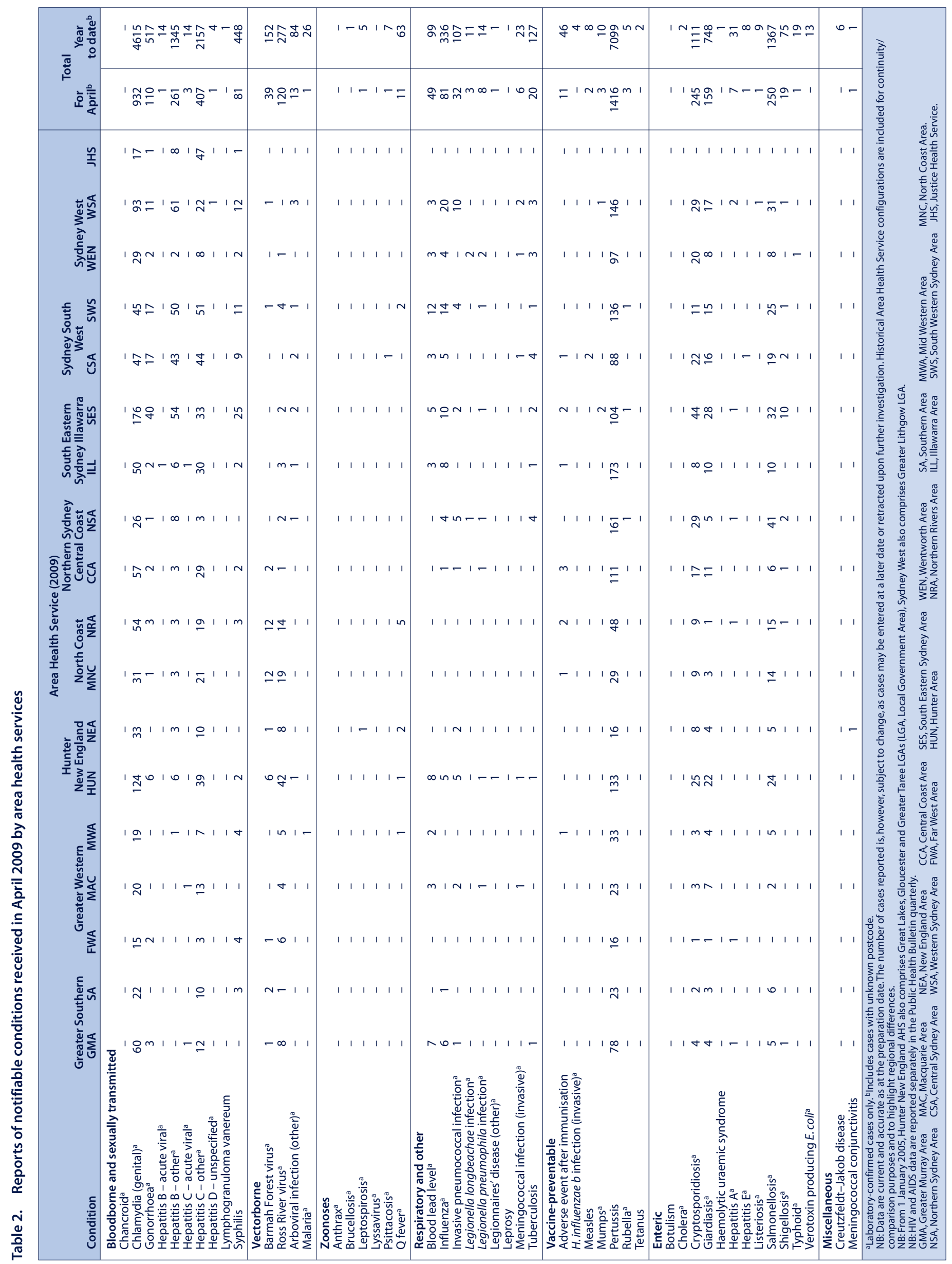

